\title{
Balanza comercial de los combustibles líquidos derivados del petróleo mediante dinámica de sistemas y simulación
}

Juan Manuel Machuca de Pina, José Antonio Taquía Gutiérrez

Ingeniería Industrial n. 27, 2009, ISSN 1025-9929, pp. 61-79

RESUMEN: El alza del precio del petróleo en los últimos años ha generado que la balanza comercial peruana de combustibles líquidos derivados del petróleo influya en la economía nacional. Debido a las múltiples variables interrelacionadas, la dinámica de sistemas y la simulación sirven como herramientas para la construcción de modelos que permiten el pronóstico de la balanza comercial.

Palabras clave: balanza comercial / Perú / combustibles / dinámica de sistemas / simulación

\section{Trade balance projection for liquid fuels derived using system dynamics and simulation}

ABSTRACT: The increment in the crude oil's price in the recent years has provoked that the Peruvian balance of trade of liquefied fuels derived from the crude oil has impact on the national economy. Due to the multiple interrelated variables, the systems dynamic and the simulation are used as tools to build patterns that help to predict the trade balance.

Key words: balance of trade / Peru / fuel / system dynamics / simulation 


\section{INTRODUCCIÓN}

Es conocido que el Perú es un país productor de petróleo, pero lo que poco se conoce es que no genera lo suficiente como para satisfacer la demanda interna de combustibles blancos, en especial de gasolina y diésel. Por ello, nos vemos afectados por la inestabilidad del precio del petróleo en el mercado mundial, que en los últimos años presenta una tendencia creciente al alza, debido principalmente al crecimiento asiático.

A finales del 2007, China tenía un 8,9\% de participación en el mercado de consumo mundial de crudo, con un crecimiento sostenido de su demanda desde el 2000, que aumentó cerca del 50\% en el periodo 20012005, y desde el 2006 presenta un promedio de crecimiento de 6\% anual, de acuerdo con las estadísticas de la Organización de Países Exportadores de Petróleo (OPEP). ${ }^{1}$ El crecimiento de este gigante asiático supera ampliamente al 7\% de crecimiento que experimentó el consumo mundial para el mismo periodo 2001-2005 y al $2 \%$ de Latinoamérica.

Esta coyuntura internacional ha generado que el gobierno destine y presupueste millones de dólares para estabilizar el fondo de compensación relacionado con el precio de los combustibles, con el fin de evitar cualquier incremento desmedido de los derivados del crudo y que esto produzca, a su vez, un impacto negativo en la canasta familiar, con la consecuente repercusión en la economía nacional. En nuestro país las reducciones de precio internacional se reflejan raramente en el precio final al consumidor, debido a que se tiene una larga cadena comercial de suministro de hidrocarburos, conformada por una gran cantidad de agentes que diluyen las disminuciones en el precio. $\mathrm{Al}$ cierre del presente artículo se mantenía la expectativa ciudadana por percibir el impacto de la reducción de precios del barril de crudo en los precios de los combustibles.

Los principales agentes que participan en esta cadena son el productor de crudo, el transportista marítimo, el operador de oleoducto, el refinador, la planta de abastecimiento, el comercializador mayorista, el transportista terrestre, el comercializador minorista, el grifo, la planta de envasado de GLP, el local de venta y, finalmente, el consumidor directo.

1 OPEC. Annual Statistical Bulletin 2007. Austria, 2008.

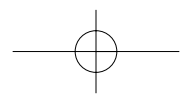


Dentro de esta larga cadena de suministro no existen facilidades para la entrada de nuevos participantes en cada uno de los sectores que la conforman. Las normas legales están estructuradas con márgenes rígidos, además de la regulación y supervisión del Ministerio de Energía y Minas (Minem) a través de la Dirección General de Hidrocarburos (DGH) y del Organismo de Supervisión de Inversión en Energía y Minería (Osinergmin). Toda nueva empresa interesada en invertir en uno de los sectores de la cadena, debe contar primero con autorizaciones que son difíciles de conseguir y de plazos largos. A ello se añade la obligación de cumplir con requisitos legales más exigentes que en otros sectores productivos.

El modelo económico tradicional nos indica que el precio es determinado por el equilibrio entre demanda y oferta. Se conoce que existen factores involucrados en el sistema, los cuales se interrelacionan e intervienen en el mercado de abastecimiento de hidrocarburos. Algunos de estos factores son, por ejemplo, la capacidad productiva actual, el abastecimiento de gas natural, el PBI, el precio internacional y los impuestos, factores cuya influencia en la demanda no ha sido estudiada.

\section{BALANZA COMERCIAL}

La balanza comercial de hidrocarburos es definida como la diferencia entre el total de las exportaciones menos el total de las importaciones de hidrocarburos que se llevan a cabo en el país. Si la balanza comercial es positiva o muestra superávit implica que son más los recursos que entran al país por las ganancias de las exportaciones que los que salen por el pago de las importaciones. Una balanza positiva incentiva y desarrolla la economía nacional. Por el contrario, una balanza comercial negativa o en déficit implica que son más los recursos que salen por las importaciones que los que ingresan por las exportaciones. Es importante, entonces, entender cómo funciona la balanza comercial analizando el contexto para identificar variables que puedan ayudar a identificar su comportamiento.

El año 2006, los autores realizamos una investigación ${ }^{2}$ que se centró principalmente en comprender la balanza comercial de los hidrocarbu-

2 Machuca de Pina, Juan M. y José A. Taquía Gutiérrez. "Proyección de la balanza comercial de los combustibles líquidos derivados del petróleo mediante dinámica de sistemas". Inédito. Universidad de Lima, Instituto de Investigación Científica, 2006. 
ros líquidos, que son compuestos bioquímicos formados únicamente por carbono e hidrógeno obtenidos a partir del petróleo, mediante destilación o por separación del gas natural. Esto incluye combustibles, lubricantes y solventes. Las principales fuentes de información en las que se basa el estudio son los organismos públicos nacionales o internacionales, que de una u otra manera intervienen en el sector: el Minem, el Osinergmin, el Instituto Nacional de Estadística e Informática (INEI), la empresa estatal Petróleos del Perú S.A. (Petroperú S.A.) y Perupetro S.A. en el ámbito nacional, y en el ámbito internacional la OPEP y la Evaluación del Impacto Ambiental (EIA) del Departamento de Energía del Gobierno de Estados Unidos, entre otros.

Antes de continuar con la explicación de la balanza comercial de hidrocarburos líquidos, debemos conocer el comportamiento de la demanda nacional de combustibles, la cual está orientada principalmente al consumo de Diésel 2 y de las gasolinas, mientras que la oferta o producción tiene un alto contenido de petróleos residuales, como podemos observar en las tablas 1 y 2 .

Tabla 1

Cuadro comparativo de ventas promedio de refinados 2000-2005 (MBD)

\begin{tabular}{lrrrrrr}
\hline & $\mathbf{2 0 0 0}$ & $\mathbf{2 0 0 1}$ & $\mathbf{2 0 0 2}$ & $\mathbf{2 0 0 3}$ & $\mathbf{2 0 0 4}$ & $\mathbf{2 0 0 5}$ \\
\hline GLP & 13,1 & 13,9 & 16,0 & 17,1 & 17,8 & 21,4 \\
Gasolina 97 & 1,9 & 1,8 & 1,9 & 1,7 & 1,6 & 1,1 \\
Gasolina 95 & 1,6 & 1,3 & 1,5 & 1,3 & 1,2 & 1,1 \\
Gasolina 90 & 7,5 & 7,7 & 7,8 & 7,8 & 8,0 & 7,0 \\
Gasolina 84 & 14,4 & 12,2 & 12,0 & 11,0 & 11,0 & 10,8 \\
Turbo & 7,3 & 7,4 & 4,3 & 3,0 & 2,9 & 2,0 \\
Kerosene & 13,8 & 15,0 & 17,6 & 11,8 & 6,9 & 4,5 \\
Diésel 2 & 54,9 & 50,0 & 49,2 & 54,3 & 63,7 & 58,6 \\
Residual 6 & 10,3 & 9,1 & 10,6 & 9,7 & 11,1 & 8,1 \\
Residual 500 & 16,3 & 13,7 & 13,4 & 12,5 & 15,9 & 15,5 \\
Otros & 6,0 & 7,1 & 5,8 & 4,5 & 4,7 & 4,1 \\
Total & 147,4 & 139,2 & 140,1 & 134,7 & 144,9 & 134,2 \\
\hline
\end{tabular}

Fuente: Ministerio de Energía y Minas. Estadística de Hidrocarburos. Elaboración propia. 
Tabla 2

Cuadro comparativo de productos refinados 2000-2005 (MBD)

\begin{tabular}{lrrrrrr}
\hline & $\mathbf{2 0 0 0}$ & $\mathbf{2 0 0 1}$ & $\mathbf{2 0 0 2}$ & $\mathbf{2 0 0 3}$ & $\mathbf{2 0 0 4}$ & $\mathbf{2 0 0 5}$ \\
\hline GLP & 6,8 & 6,3 & 6,7 & 6,7 & 7,3 & 7,3 \\
Gasolina 97 & 2,4 & 2,0 & 2,2 & 2,2 & 2,0 & 1,4 \\
Gasolina 95 & 1,5 & 1,4 & 1,5 & 1,4 & 1,3 & 1,1 \\
Gasolina 90 & 7,6 & 8,0 & 7,7 & 7,8 & 8,2 & 7,5 \\
Gasolina 84 & 13,8 & 12,2 & 11,5 & 11,6 & 10,9 & 10,3 \\
Kerosene & 14,3 & 14,9 & 15,0 & 10,8 & 7,0 & 5,0 \\
Solvente 1 & 0,5 & 0,5 & 0,9 & 1,3 & 1,1 & 0,9 \\
Solvente 3 & 0,4 & 0,4 & 0,4 & 0,5 & 0,6 & 0,7 \\
Otros solventes & 0,0 & 0,0 & 0,1 & 0,0 & 0,0 & 0,1 \\
Otras gasolinas & 1,0 & 0,0 & 0,6 & 3,4 & 1,8 & 7,0 \\
Turbo A-1 & 7,7 & 7,7 & 8,0 & 8,6 & 9,3 & 9,3 \\
Diésel 2 & 33,8 & 36,9 & 37,5 & 38,0 & 40,0 & 45,9 \\
IFO & 0,5 & 0,4 & 1,6 & 2,6 & 2,9 & 2,9 \\
Residual 5 & 0,0 & 0,1 & 0,1 & 0,4 & 0,1 & 0,3 \\
Residual 6 & 18,3 & 18,2 & 18,9 & 18,3 & 17,7 & 13,8 \\
Residual 500 & 32,0 & 34,6 & 29,2 & 30,2 & 30,0 & 34,6 \\
Asfalto líquido & 0,5 & 0,5 & 0,5 & 0,4 & 0,6 & 0,5 \\
Naftas (Naftoil+Naftas) & 6,0 & 8,1 & 6,4 & 5,1 & 11,0 & 11,4 \\
Otros & 7,3 & 8,7 & 11,2 & 12,3 & 12,8 & 20,6 \\
Total & 154,4 & 161,0 & 160,0 & 161,6 & 164,3 & 180,7 \\
\hline
\end{tabular}

Fuente: Ministerio de Energía y Minas. Estadística de Hidrocarburos.

Elaboración propia.

Las características del crudo peruano y de las instalaciones nacionales hacen que el consumo dependa de la importación de hidrocarburos líquidos y, por tanto, esté sujeto a las variables del mercado internacional. En diciembre del 2005, el volumen total de las importaciones de crudo y refinados equivalía al $153 \%$ del volumen de crudo extraído.

En las tablas 3 y 4 puede apreciarse el resumen volumétrico anual de importaciones y exportaciones de crudo y refinados de petróleo, componentes principales de la balanza comercial de hidrocarburos líquidos. 
Tabla 3

Exportaciones FOB de hidrocarburos líquidos 2000-2005 (MBLS)

\begin{tabular}{|c|c|c|c|c|c|c|}
\hline & 2000 & 2001 & 2002 & 2003 & 2004 & 2005 \\
\hline Crudo & 4.246 & 6.434 & 7.819 & 11.008 & 6.083 & 4.348 \\
\hline Gases & 0 & 0 & 0 & 0 & 494 & 1.556 \\
\hline GLP & & & & & 17 & 0 \\
\hline Butano & & & & & 181 & 531 \\
\hline Propano & & & & & 296 & 1.024 \\
\hline Gasolinas & 1.956 & 2.166 & 2.296 & 2.228 & 4.835 & 9.094 \\
\hline Nafta virgen/ nafta alto & 1.397 & 1.815 & 2.254 & 1.743 & 3.328 & 8.000 \\
\hline Gasolina motor + natural & 559 & 351 & 43 & 485 & 1.507 & 1.094 \\
\hline Destilados medios & 1.027 & 977 & 1.774 & 1.949 & 2.010 & 4.045 \\
\hline Kero turbo & & 578 & 1412 & 129 & & \\
\hline Turbo A-1 & 337 & & & 1.465 & 1.585 & 2.378 \\
\hline Turbo JP-5 & 21 & 0 & 2 & 6 & 7 & 1 \\
\hline Diésel 2 & 669 & 398 & 280 & 348 & 419 & 509 \\
\hline Material de corte/ gasoils & & & 80 & & & 1.156 \\
\hline Residuales & 5.960 & 7.708 & 8.909 & 9.112 & 8.378 & 8.653 \\
\hline \multicolumn{7}{|l|}{ Crudo reducido } \\
\hline Residual 6 & 1.146 & 1.202 & 2.488 & 2.153 & 2.162 & 1.858 \\
\hline Residual 500 & 4.268 & 5.507 & 6.067 & 6.576 & 5.365 & \\
\hline IFO Interm.Fuel Oil/ MGO & 546 & 999 & 355 & 383 & 851 & 6795 \\
\hline Otros & 1 & 1 & 2 & 16 & 33 & 21 \\
\hline \multicolumn{7}{|l|}{ Asfaltos } \\
\hline Acido nafténico & 0 & 1 & 1 & 0 & 2 & \\
\hline Solvente 1 & & & & & 24 & 19 \\
\hline Solvente 3 & & & & & 7 & 2 \\
\hline Total & 13.189 & 17.286 & 20.801 & 24.313 & 21.834 & 27.716 \\
\hline
\end{tabular}

Fuente: Ministerio de Energía y Minas. Estadística de Hidrocarburos. Elaboración propia. 
Tabla 4

Importaciones CIF de hidrocarburos líquidos 2000-2005 (MBLS)

\begin{tabular}{lrrrrrr}
\hline & $\mathbf{2 0 0 0}$ & $\mathbf{2 0 0 1}$ & $\mathbf{2 0 0 2}$ & $\mathbf{2 0 0 3}$ & $\mathbf{2 0 0 4}$ & $\mathbf{2 0 0 5}$ \\
\hline Crudo & $\mathbf{1 8 . 1 7 1}$ & $\mathbf{2 3 . 9 7 0}$ & $\mathbf{2 6 . 9 3 5}$ & $\mathbf{2 9 . 3 8 5}$ & $\mathbf{3 0 . 3 0 8}$ & $\mathbf{3 4 . 9 9 0}$ \\
Gasolinas & $\mathbf{4 4 5}$ & $\mathbf{3 8 0}$ & $\mathbf{4 1 0}$ & $\mathbf{3 1 1}$ & $\mathbf{3 6 2}$ & $\mathbf{8 3 9}$ \\
Gasolina de aviación & 0 & 39 & 16 & 5 & 11 & 10 \\
Nafta reformada (HGBS) & 445 & 342 & 366 & 306 & 325 & 82 \\
Gasolina motor + natural & 0 & 0 & 27 & 0 & 25 & 747 \\
Destilados medios & $\mathbf{8 . 4 1 4}$ & $\mathbf{5 . 7 7 3}$ & 7.023 & $\mathbf{9 . 9 0 6}$ & $\mathbf{9 . 8 1 1}$ & $\mathbf{6 . 0 1 6}$ \\
Keroturbo & 1.110 & 269 & 1.487 & 1.188 & 1 & 0 \\
Diésel & 7.046 & 5.354 & 4.974 & 7.079 & 9.061 & 5.779 \\
Material de corte & 258 & 150 & 563 & 1639 & 748 & 237 \\
GLP & $\mathbf{5 1}$ & $\mathbf{3 9 2}$ & $\mathbf{9 8 0}$ & $\mathbf{8 9 0}$ & $\mathbf{2 8 7}$ & $\mathbf{1 6}$ \\
Butano & $\mathbf{6 2 8}$ & $\mathbf{4 7 1}$ & $\mathbf{4 9 8}$ & $\mathbf{7 1 7}$ & $\mathbf{7 8 2}$ & $\mathbf{2 6 4}$ \\
Propano & $\mathbf{1 . 0 5 3}$ & $\mathbf{1 . 2 9 9}$ & $\mathbf{1 . 3 8 4}$ & $\mathbf{1 . 8 7 1}$ & $\mathbf{1 . 6 9 3}$ & $\mathbf{4 5 6}$ \\
Otros & $\mathbf{4}$ & $\mathbf{3}$ & $\mathbf{5}$ & $\mathbf{8 6}$ & $\mathbf{3 4}$ & $\mathbf{3 2 4}$ \\
Solventes & 4 & 3 & 5 & 70 & 9 & 302 \\
MTBE + Comb. industrial & 0 & 0 & 0 & 15 & 26 & 22 \\
Prod. Petroquímicos & $\mathbf{0}$ & $\mathbf{0}$ & $\mathbf{0}$ & $\mathbf{1 9 1}$ & $\mathbf{0}$ & $\mathbf{0}$ \\
Total & $\mathbf{2 8 . 7 6 5}$ & $\mathbf{3 2 . 2 8 3}$ & $\mathbf{3 7 . 2 3 5}$ & $\mathbf{4 3 . 3 5 7}$ & $\mathbf{4 3 . 2 7 8}$ & $\mathbf{4 2 . 9 0 4}$ \\
\hline
\end{tabular}

Fuente: Ministerio de Energía y Minas. Estadística de Hidrocarburos. Elaboración propia.

La balanza comercial peruana de hidrocarburos ha venido mostrando un continuo deterioro, debido principalmente al aumento sostenido de las importaciones. Mientras que en 1982 se importaba 1.600 barriles por día (BD), en el 2004 se importó un total de $123.300 \mathrm{BD}$, registrándose un déficit de US $\$ 1.029$ millones. Por otro lado, las exportaciones, en cuanto a volumen, llegaron a su pico más alto en 1985, y desde entonces se han mantenido prácticamente estancadas. Esto ha hecho que, en términos monetarios, la balanza comercial agregada sea deficitaria desde $1988 .^{3}$

\section{SECTOR ENERGÉTICO}

Ahora bien, considerando que la producción de energía nacional obtenida a partir del petróleo en el año 2004 fue de 38,8\% — cifra que llega

3 Energy Information Administration. "Internacional Energy Annual 2003. Internal EIA estimates” [en línea]. <www.eia.doe.gov>. [Consulta: noviembre del 2008.] 
a alcanzar un $51 \%$ si se tiene en cuenta solo la producción comercial de energía, como puede apreciarse en la tabla 5 -, podemos entonces observar que la producción nacional depende principalmente del petróleo.

Tabla 5

Producción y reservas probadas de energía comercial 2004 (TJ)

\begin{tabular}{lrrrr}
\hline Fuente & Producción & Reservas & \multicolumn{2}{c}{ Estructuras } \\
& & & Producción & Reservas \\
\hline Petróleo & 169.338 & 2.196 .486 & 51,0 & 8,8 \\
Hidroenergía & 98.532 & 5.965 .666 & 29,7 & 24,0 \\
Gas natural + LGN & 63.367 & 15.048 .491 & 19,1 & 60,4 \\
Carbón mineral $\left(^{*}\right)$ & 652 & 1.700 .811 & 0,2 & 6,8 \\
Total & 331.889 & 24.911 .454 & 100,0 & 100,0 \\
\hline
\end{tabular}

Fuente: Minem. Balance nacional de energía 2004, p. 9.

Por ello, el Estado peruano impulsa la transformación de la matriz energética hacia el consumo de sustitutos como el gas natural, ya que si bien hay algunos proyectos para la producción de biodiésel o metanol, en general existe poca oferta de sustitutos a los combustibles derivados del petróleo, por lo que el mercado está supeditado a las políticas empresariales y de precios de los dos principales refinadores nacionales: Repsol-YPF y Petroperú, que controlan el 100\% de la refinación procedente del petróleo y cerca del $70 \%$ de la producción de hidrocarburos, ${ }^{4}$ incluyendo los líquidos extraídos de los reservorios de gas natural.

El sustituto natural del petróleo es el gas natural, cuyos fluidos se extraen del subsuelo y son una mezcla de gas natural y líquidos de gas natural (LGN). Actualmente se encuentra en producción en las plantas de Aguaytía, Pariñas y Camisea, siendo Pluspetrol el principal productor. Los fluidos son separados y el LGN es conducido a las plantas de fraccionamiento ubicadas en Pisco (en el caso de Camisea) y Pucallpa (en el caso de Aguaytía).

4 Osinergmin. "Producción fiscalizada de hidrocarburos líquidos". Noviembre 2008 [en línea]. <www.osinerg.gob.pe>. [Consulta: diciembre del 2008.] 
La producción de gas natural y sus derivados líquidos de Camisea está destinada a generar un efecto de sustitución de consumo en los sectores industrial, transporte y comercio, residencial y exportación, para reducir la dependencia de las importaciones a través del efecto combinado de producción de líquidos, sustitución de consumo y exportación y consumo de gas natural y gas natural licuado. Sin embargo, ni las empresas productoras de gas natural ni las empresas afectadas por la sustitución creían que esto fuera a producirse en forma rápida; ya han transcurrido varios años desde que se inició el proceso y las importaciones continúan creciendo; el reporte de importaciones CIF de octubre del 2008 fue de 5.298 MBLS. $^{5}$

Las principales empresas del sector industrial ya han efectuado la sustitución; sin embargo, el predominio de pequeñas empresas dificultan la conversión. En el sector transporte, la sustitución de consumo solo se da de manera significativa en el subsector de transporte público, el cual durante la década de 1990 había sido inducido hacia el consumo de Diésel-2, haciendo imposible la migración directa de diésel a gas por razones tecnológicas, por lo que el efecto de sustitución se irá produciendo en la medida en que el parque automotor se vaya renovando. En el sector residencial, las características de geografía y latitud del territorio peruano hacen que no existan cambios climatológicos radicales con inviernos crudos que requieran un fuerte consumo energético en calefacción, por ejemplo. Es por esta razón que la empresa productora ha apostado mayormente por la exportación de gas natural y la extracción de sus líquidos asociados.

En este momento, los líquidos asociados al gas natural representan alrededor del 30\% de la producción nacional de líquidos. Estos líquidos tienen un alto valor comercial y son vendidos con mayor facilidad que el gas natural. Entonces, al no existir suficiente demanda de gas seco, actualmente se opta por reinyectarlo a los pozos para poder comercializar los líquidos asociados. La producción de líquidos derivados del gas de Camisea no disminuirá el déficit de líquidos a corto plazo, aunque su entrada sí ha modificado la tendencia para algunos productos como el GLP, pasando de ser importadores a ser exportadores, pero cuyo volumen no es significativo aún en la balanza comercial.

5 Minem. "Balanza comercial octubre 2008" [en línea]. <www.minem.gob.pe>. [Consulta: diciembre del 2008.] 


\section{MODELOS PARA PRONÓSTICO DE LA BALANZA COMERCIAL}

Dado que existen múltiples variables que pueden afectar el comportamiento de la balanza comercial, las cuales se relacionan y retroalimentan entre sí, es necesaria una herramienta que permita explicar y proyectar su comportamiento de manera dinámica. Todo modelo dinámico se inicia al dar respuesta a las preguntas relacionadas con lo que se pretende obtener del modelo. Distintos enfoques sobre modelamiento de procesos se orientan en función a la aplicación, al tratamiento de los datos disponibles y al uso que se desea dar a la información obtenida. Independientemente de estos enfoques, el principio que hay que respetar es la eficacia en identificar el nivel de detalle que se va a representar para evitar omisiones serias o excederse en el nivel de complejidad del modelo que se quiere obtener. Para ello se ha utilizado la dinámica de sistemas.

$\mathrm{Al}$ representar adecuadamente un modelo en dinámica de sistemas, se tienen que tomar en consideración las demoras de tiempo, la amplificación y la distorsión de la información. Todas las constantes y variables del modelo pueden y deben ser equivalentes a las cantidades y conceptos correspondientes en el sistema real. Las unidades dimensionales de medida de las cantidades del modelo tienen que ser consistentes. Una de las prácticas más usadas es comenzar con la estructura continua (no estocástica) de las decisiones del sistema y, posteriormente, agregar el azar y las influencias periódicas.

Los métodos de formulación de modelos no deben presuponer el carácter lineal o la estabilidad del sistema. Cualquier modelo ha de incorporar una estructura matemática adecuada que represente un sistema dinámico.

El diagrama de Forrester que se presenta en el gráfico 1 describe el modelo propuesto, que representa el comportamiento de la cantidad de crudo y refinados que produce el país y las necesidades de importación y las exportaciones. Las variables consideradas en él son: balanza comercial, exportación, importación total, importación de productos terminados, periodo de tiempo, inventario de productos, producción de refinados y ventas. 
Balanza comercial de los combustibles líquidos derivados del petróleo
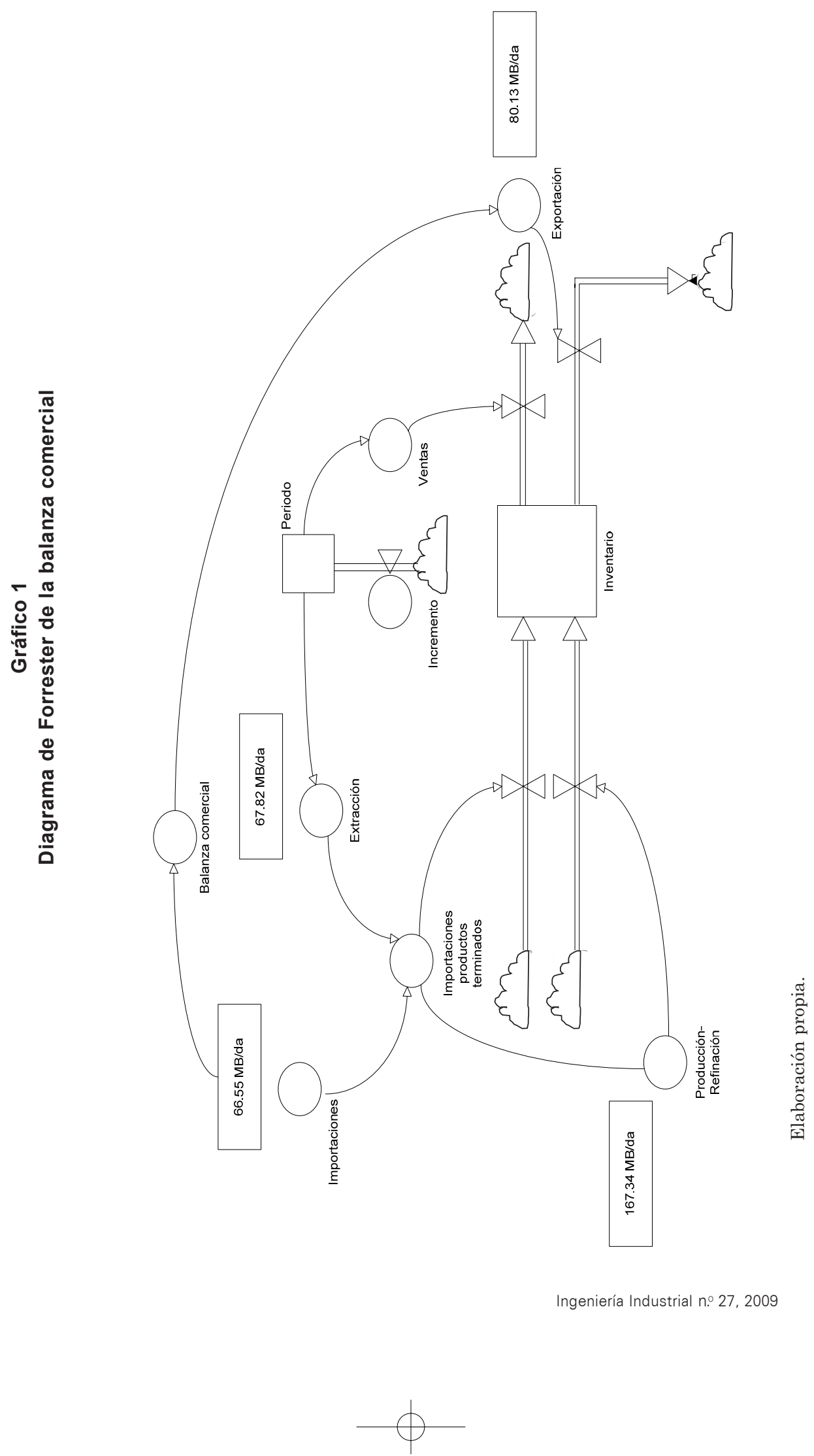
Para cuantificar las variables e interrelaciones del modelo dinámico se ha utilizado el software Powersim, tal como se muestra en el gráfico 2.

\section{Gráfico 2}

\section{Ecuaciones del modelo en Powersim}

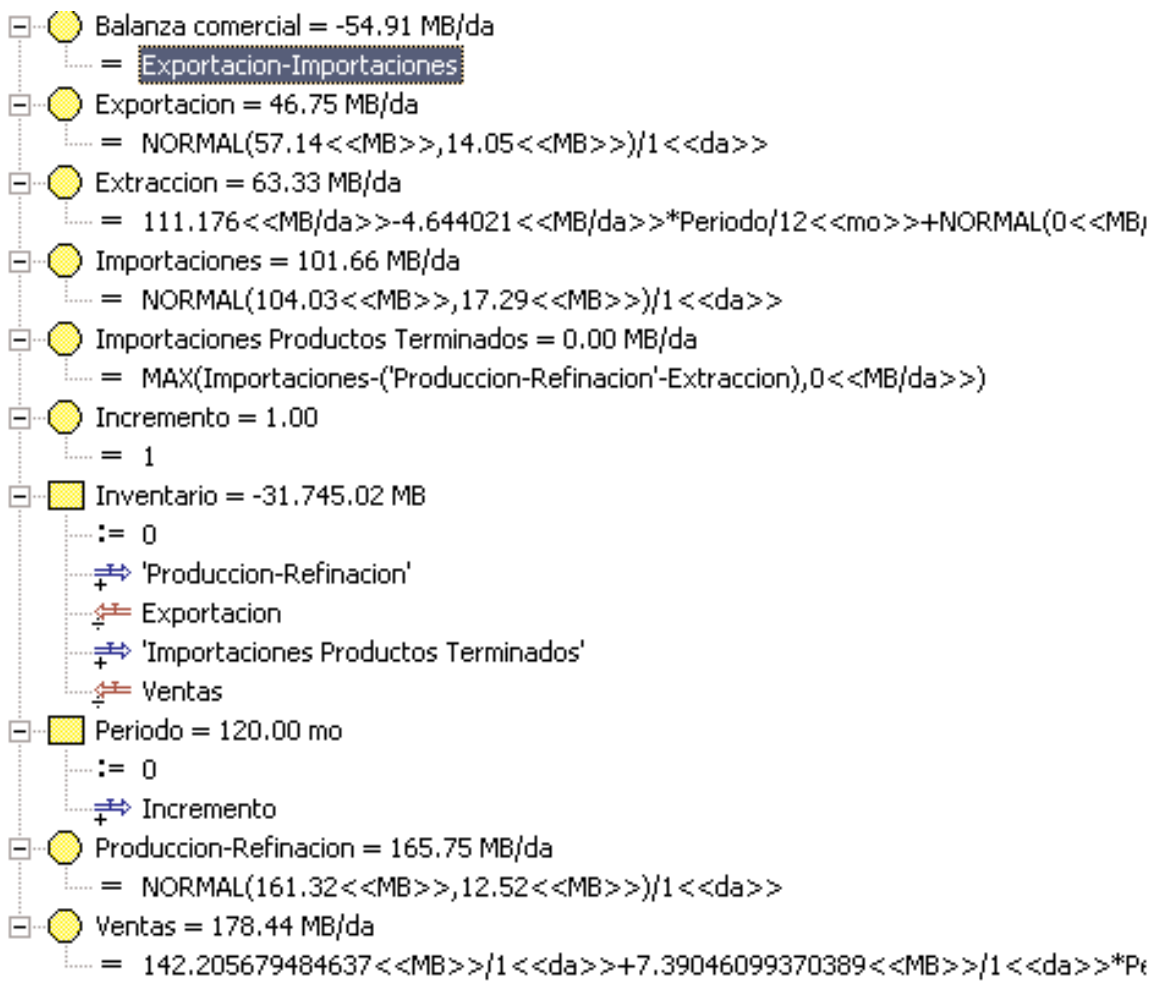

Elaboración propia.

Para obtener los coeficientes de las variables utilizadas en las ecuaciones matemáticas del modelo dinámico, se propone un modelo matemático de programación no lineal, cuya función final es minimizar el error de los valores generados por el modelo utilizando series de datos históricos que se obtuvieron en la investigación que los autores realizamos el 2006, suavizados cuando los comportamientos presentaron picos extremos en su comportamiento. Los resultados son contrastados con los datos históricos para verificar la validez de la solu- 
ción propuesta. El modelo no lineal se resolvió utilizando el software Lingo, el cual se puede ver en el gráfico 3 y los resultados se aprecian en el gráfico 4.

Gráfico 3

Modelo no lineal regresivo

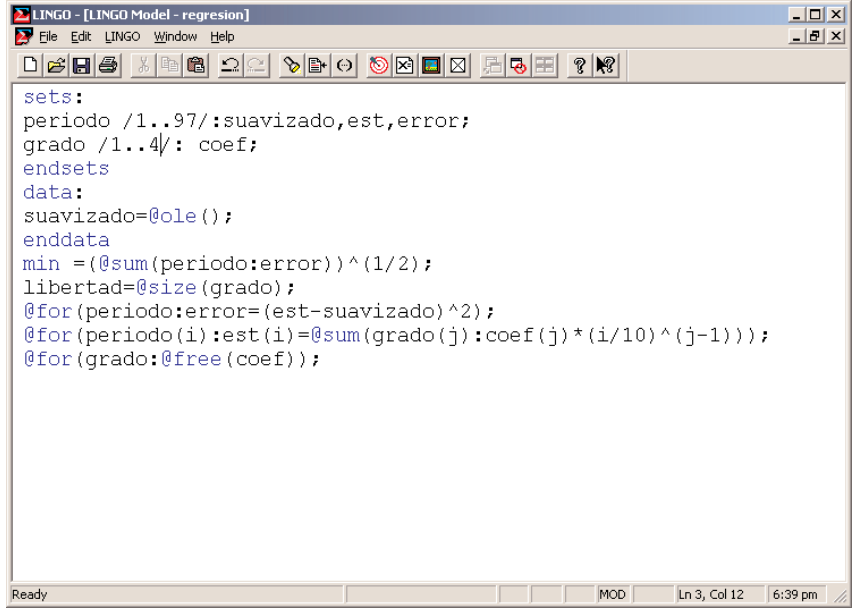

Elaboración propia.

Gráfico 4

Resultados del modelo no lineal regresivo

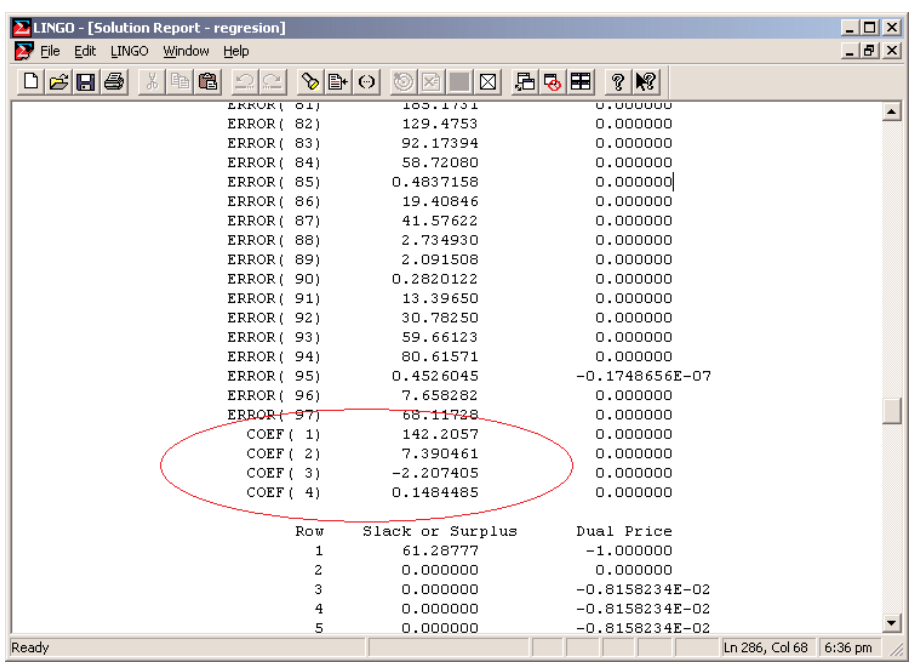

Elaboración propia. 
El software Powersim permite obtener como resultados curvas que representan el comportamiento de las variables que participan en el modelo propuesto. En los gráficos 5 y 6 se muestran algunas de las principales variables del modelo.

Gráfico 5

Pronóstico de producción vs. ventas de combustibles líquidos

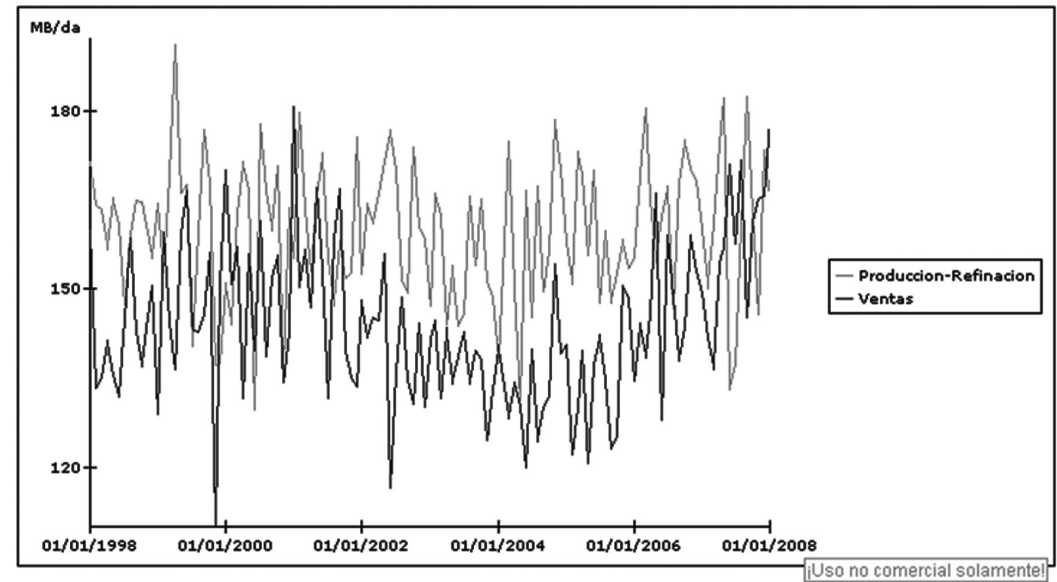

Elaboración propia.

Gráfico 6

Pronóstico de la balanza comercial de combustibles líquidos

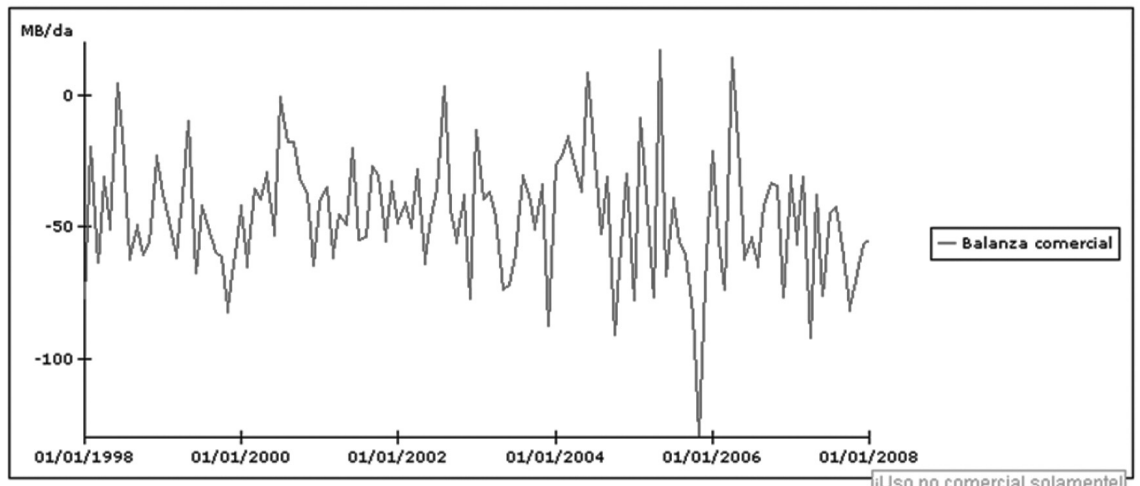

Elaboración propia. 
Dado el interés por conocer el funcionamiento de los agentes que participan en la cadena de suministro, se propuso un modelo de simulación para representar el proceso de importación y comercialización del crudo, desde que llega a los terminales de almacenamiento hasta que se traslada a los grifos para su comercialización.

El modelo de simulación toma en cuenta los agentes existentes en la cadena comercial con sus respectivos ratios y flujos que generan ritmos de demanda por hora. Los ratios para fines de estudio son aleatorios, obteniéndose de una distribución normal que tiene por media el pronóstico vigente y como desviación estándar al $20 \%$ de la media. La demanda de combustible se realiza en forma continua las 24 horas del día, los siete días de la semana, lo mismo que su distribución, producción y abastecimiento. La planta puede atender a un número variable de grifos, parámetro del modelo, cada uno de los cuales se comporta de forma independiente con su respectivo submodelo gracias a las facilidades del lenguaje de programación de la herramienta de simulación Arena 10.0.

Gráfico 7

Modelo de simulación de trabajo de refinería

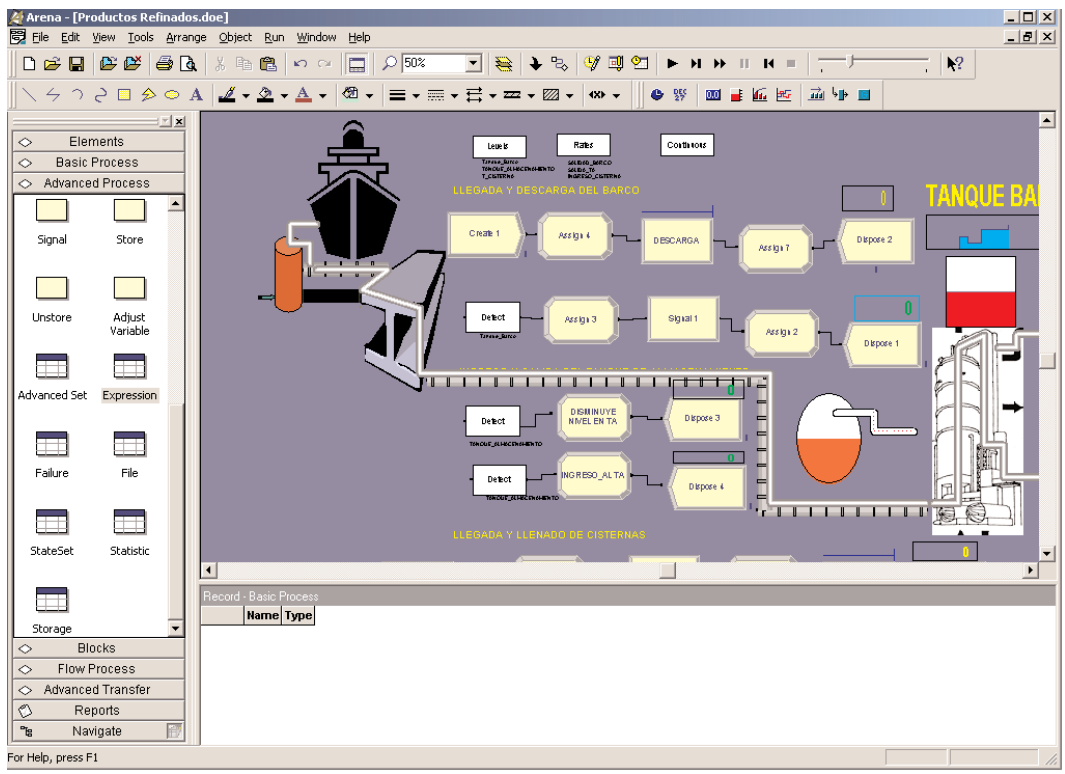




\section{Gráfico 8}

Detalle de ingreso de cantidad de grifos

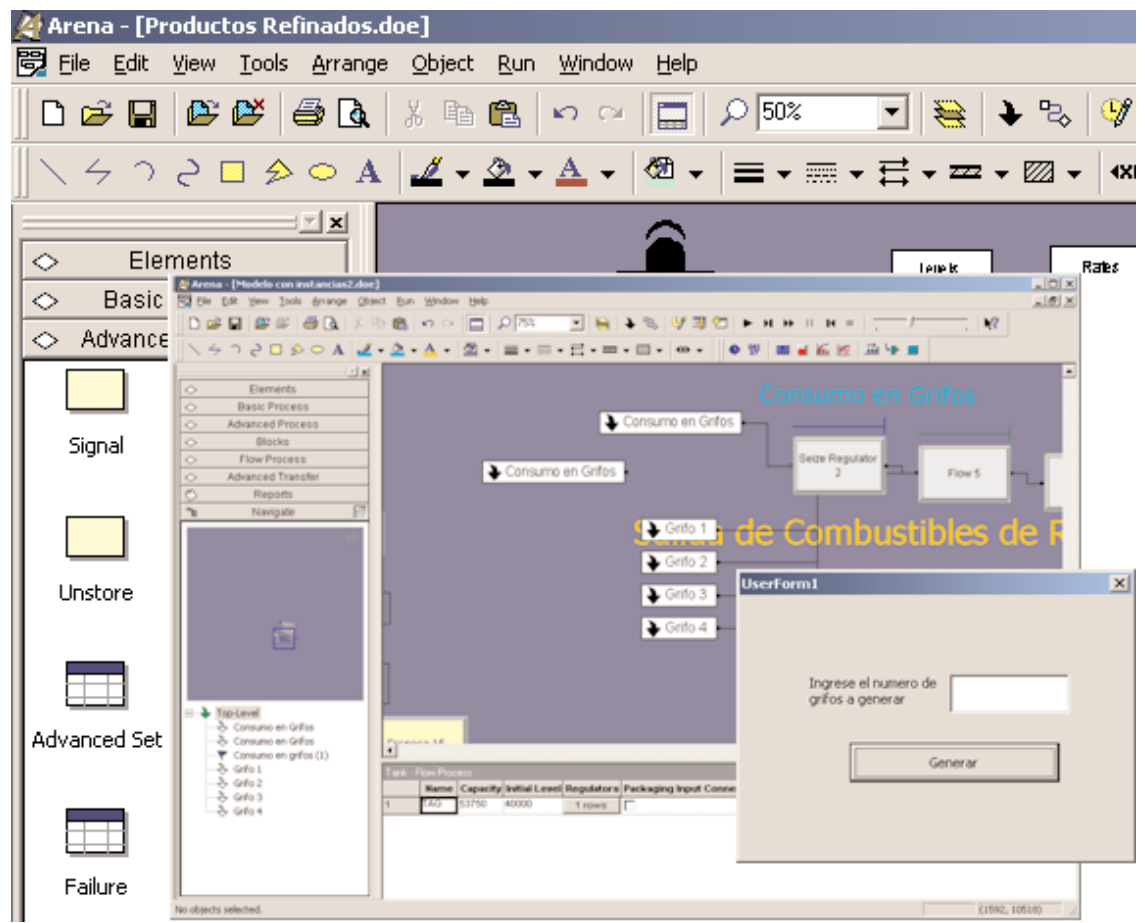

Según el detalle en el punto final de la cadena de suministro, el funcionamiento de la estación de servicio (grifo) responde a la cantidad de surtidores con que cuenta una estación mediana con seis tanques de almacenamiento de combustible y cuatro surtidores (distribuidos en dos zonas) que tienen seis pistolas cada uno para el despacho de combustible al cliente. Por día existen dos turnos: mañana ( 7 a.m.) y noche (7 p.m.). En cada surtidor se puede atender, como máximo, dos vehículos, y en toda la estación se presta servicios simultáneamente a un máximo de ocho; pueden esperar solo cuatro vehículos a que se desocupe un surtidor. Si un cliente llega y encuentra la estación en su capacidad total, se retira. El llenado de los tanques no interfiere la atención en los surtidores, en la plataforma de los tanques solo una cisterna puede realizar la descarga; las demás tienen que esperar. 
Tabla 6

Detalle de parámetros de consumo promedio en grifo

\begin{tabular}{cccc}
\hline Tipo de gasolina & $\begin{array}{c}\text { Stock seguridad } \\
\text { (gal) }\end{array}$ & $\begin{array}{c}\text { Punto de orden } \\
\text { (gal) }\end{array}$ & $\begin{array}{c}\text { Orden de compra } \\
\text { reabastecimiento }\end{array}$ \\
\hline 97 & 100 & 200 & 1.000 \\
95 & 100 & 200 & 1.000 \\
90 & 100 & 300 & 3.000 \\
84 & 100 & 250 & 2.000 \\
D2 & 200 & 400 & 5.000 \\
D1 & 100 & 200 & 1.000 \\
\hline
\end{tabular}

Elaboración propia.

El modelo considera demoras en el tránsito a la planta, la atención y el retorno, todas con distribución normal. Asimismo, se estableció que el tiempo entre arribos de los clientes responde a una distribución exponencial con valor medio que varía según el tipo de hora y de día. Además, considera el trabajo de atención para aproximadamente 1.5000 autos en 15 días, y se debe mencionar también que el sistema modelado es del tipo sistema de estado estable pero con replicaciones truncadas. Se establecen, en efecto, dos diferentes situaciones: fin de semana y día entre semana, considerando horas punta, porque el comportamiento del tiempo entre arribos de los autos varía en función del día y la hora. Se ha establecido un tiempo de calentamiento de cinco horas para una longitud de simulación de quince días, utilizando la variable cantidad de vehículos en la estación de servicio, tiempo medio de atención y longitud promedio de cola.

Para determinar el comportamiento de la producción de los diferentes productos en el período estudiado entre los años 2000-2005 se utilizó el software Input Analyzer. 10.0 de Rockwell Software, que realiza pruebas de bondad de ajuste (Prueba Chi Cuadrado y Kolgomorov Smirnov). Como resultado de estas pruebas estadísticas, se determinó que el comportamiento de la demanda de los productos refinados responde a un comportamiento normal, que es el utilizado en el modelo de simulación para representar el consumo de combustible. 


\section{RESULTADOS DE LOS MODELOS}

El modelo de simulación permitirá ver los flujos entre los agentes que participan en la cadena de suministro de combustibles derivados del petróleo, todos ellos del sector de hidrocarburos, y cómo los cambios en cada uno de los diferentes agentes pueden afectar la utilización de los recursos de las plantas de refinación.

Regresando al modelo propuesto de dinámica de sistemas, también explica y proyecta el comportamiento volumétrico de la balanza comercial de combustibles líquidos derivados de petróleo en miles de barriles por día. Los resultados numéricos del modelo dinámico propuesto mostraban que la balanza comercial de combustibles líquidos seguirá siendo negativa en volumen, y por tanto en valor expresado en moneda, mientras no se impulse la exploración de hidrocarburos y, de esta forma, se incremente la explotación, cuya producción tiene una tendencia decreciente. El otro camino para revertir la balanza comercial es mediante productos que sustituyan a los combustibles líquidos; sin embargo, no se espera que esta sustitución suceda en el corto sino en el largo plazo, ya que se destina la mayor parte del gas natural a la exportación para revertir lo negativo de la balanza comercial de hidrocarburos a positivo en valor monetario. Ello no se reflejará en el bolsillo de los ciudadanos, quienes seguirán consumiendo los productos derivados de petróleo de alto valor. El incremento de la capacidad productiva no alterará significativamente la balanza comercial, debido a la falta de extracción de crudo suficiente en calidad y cantidad para la producción de refinados. Será decisión del Estado establecer las políticas que favorezcan a cualquiera de estos dos caminos, si se pretende tener una balanza comercial positiva.

En función de los datos pronosticados, se considera que el Estado peruano debería favorecer las actividades de exploración para incrementar la producción de crudo; de esta forma se equilibraría la balanza comercial de combustibles líquidos y aumentaría la factibilidad de proyectos de inversión destinados a incrementar la capacidad de refinación.

Finalmente, debería favorecerse el consumo interno de gas natural, pues sin el incremento del consumo en servicios básicos no existirá un beneficio directo en los costos energéticos de los ciudadanos. 


\section{BIBLIOGRAFÍA}

Aracil, J. y F. Gordillo. Dinámica de sistemas. Madrid: Alianza Editorial, 1997.

Altman, Morris. Handbook of contemporary behavioral economics foundations and developments. Nueva York: Sharpe, inc., 2006.

Averill, Law y David Kelton. Simulation modeling and analysis. 3. ${ }^{\mathrm{a}}$ edición. Nueva York: McGraw- Hill, 2000.

Checkland, Peter. Pensamiento de sistemas. México: Noriega, 2003.

Delurgio, Stephen. Forecasting principles and applications. Boston: Irwin/McGraw-Hill, 1998.

Forrester, Jay. Industrial dynamics. Massachusetts: MIT Press, 1961.

Friend, Jhon y Allen Hickling. Planning under pressure. The strategic choice approach. 3. ${ }^{\mathrm{a}}$ edición. Amsterdam: Elsevier Butterworth Heinemman, 2005.

Gallardo Ku, J.; Vásquez Cordano, A. y L. Bendezú Medina. La problemática de los precios de los combustibles. Documento de Trabajo 11. Lima: Osinerg, Oficina de Estudios Económicos, 2005.

Hernández Sampieri, R.; Fernández Collado, C. y P. Baptista Lucio. Metodología de la investigación. México, D.F.: McGraw-Hill, 2003.

McGarvey, Bernard. Modeling for business management an introduction. Nueva York: Springer Verlag, 2004.

Morecroft, John D. W. Modeling for learning organizations. Nueva York: Productivity Press, 2000.

Rodríguez Ulloa, Ricardo. La sistémica, los sistemas blandos y los sistemas de información. Lima: Universidad del Pacífico, 1994.

Senge Peter. La danza del cambio. Los retos de sostener el impulso en organizaciones abiertas al aprendizaje. Bogotá: Norma, 2000.

Sterman, Jhon D. Business dynamics. Systems thinking and modeling for a complex world. Boston, MA: McGraw-Hill Higher Education Edition, 2000.

Vásquez Cordano, Arturo. "La organización económica de la industria de hidrocarburos en el Perú". Documento de Trabajo 8. Lima: Osinerg, Oficina de Estudios Económicos, 2005.

Vásquez Cordano, A.; Gallardo, J.; Bendezú, L.; Salvador, J. y F. Amésquita. "La informalidad y sus manifestaciones en la comercialización de combustibles líquidos en el Perú. Documento de Trabajo". N. ${ }^{\circ}$ 15. Lima: Osinerg, Oficina de Estudios Económicos, 2004. 